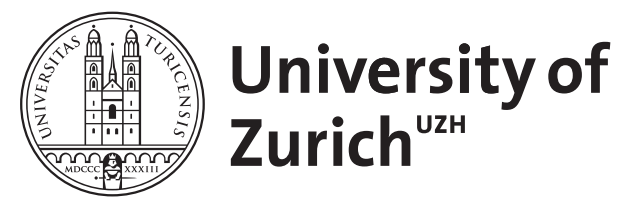

\title{
Kognitive Aktivierung im Mathematikunterricht
}

Pauli, C ; Drollinger-Vetter, B ; Hugener, I ; Lipowsky, F

\begin{abstract}
Ausgehend von einem konstruktivistischen Lernkonzept wurde die Unterrichtsqualität in 38 videografierten Unterrichtseinheiten (je 3 Mathematikstunden deutscher und schweizerischer Lehrpersonen, Sekundarstufe I, N = 773 Schüler/innen) anhand von drei für die kognitive Aktivierung der Lernenden relevanten Dimensionen erfasst und auf den Lernerfolg bezogen. Dabei wurde aufgrund des methodischen Vorgehens beim Begriffsaufbau in der Einführungsphase (fragend-entwickelndes Lehrgespräch/Lehrervortrag vs. explorativentdeckendes Vorgehen) zwischen zwei Gruppen von Unterrichtseinheiten unterschieden. Die Ergebnisse zeigen, dass die Ausprägung der drei Qualitätsmerkmale vom methodischen Vorgehen beim Begriffsaufbau unabhängig ist und dass die fachlich-inhaltliche Qualität des Unterrichts (inhaltlich-strukturelle Klarheit) sowie die Qualität des Klassengesprächs (substanzielle Schülerbeteiligung) zur Erklärung des Lernerfolgs beitragen.
\end{abstract}

DOI: https://doi.org/10.1024/1010-0652.22.2.127

Posted at the Zurich Open Repository and Archive, University of Zurich

ZORA URL: https://doi.org/10.5167/uzh-14445

Journal Article

Accepted Version

Originally published at:

Pauli, C; Drollinger-Vetter, B; Hugener, I; Lipowsky, F (2008). Kognitive Aktivierung im Mathematikunterricht. Zeitschrift für Pädagogische Psychologie, 22(2):127-133.

DOI: https://doi.org/10.1024/1010-0652.22.2.127 
Kolumnentitel: KOGNITIVE AKTIVIERUNG IM MATHEMATIKUNTERRICHT

Kognitive Aktivierung im Mathematikunterricht

Christine Pauli ${ }^{1}$, Barbara Drollinger-Vetter ${ }^{3}$, Isabelle Hugener ${ }^{1} \&$ Frank Lipowsky $^{2}$

${ }^{1}$ Universität Zürich

${ }^{2}$ Universität Kassel

${ }^{3}$ Pädagogische Hochschule Zürich

Zeitschrift für Pädagogische Psychologie, 2008, 22(2), 127-133. 


\section{Zusammenfassung}

Ausgehend von einem konstruktivistischen Lernkonzept wurde die Unterrichtsqualität in 38 videografierten Unterrichtseinheiten (je 3 Mathematikstunden deutscher und schweizerischer Lehrpersonen, Sekundarstufe I, $N=773$ Schüler/innen) anhand von drei für die kognitive Aktivierung der Lernenden relevanten Dimensionen erfasst und auf den Lernerfolg bezogen. Dabei wurde aufgrund des methodischen Vorgehens beim Begriffsaufbau in der Einführungsphase (fragend-entwickelndes Lehrgespräch/Lehrervortrag vs. explorativentdeckendes Vorgehen) zwischen zwei Gruppen von Unterrichtseinheiten unterschieden. Die Ergebnisse zeigen, dass die Ausprägung der drei Qualitätsmerkmale vom methodischen Vorgehen beim Begriffsaufbau unabhängig ist und dass die fachlich-inhaltliche Qualität des Unterrichts (inhaltlich-strukturelle Klarheit) sowie die Qualität des Klassengesprächs (substanzielle Schülerbeteiligung) zur Erklärung des Lernerfolgs beitragen.

Schlüsselwörter: Mathematikunterricht, Unterrichtsqualität, verständnisvolles Lernen 


\section{Abstract: Cognitive activation and mathematics instruction}

Based on a constructivist concept of learning, the instructional quality of videotaped teaching units ( 3 mathematics lessons in each case) of 19 German and 19 Swiss teachers $(\mathrm{N}=773$ students) from the $8^{\text {th }} / 9^{\text {th }}$ school year is examined in terms of three quality features related to cognitive activation of the learners. Teaching quality is then related to learning success. A distinction is made with respect to the procedure of conceptual construction (teacher-guided vs. discovery-oriented). The results show that instructional quality does not depend upon the method of conceptual construction within the unit, and that the quality of content-related activities (structural clarity) and the quality of classroom discourse (degree of student participation) contribute to explaining learning success.

Key words: Mathematics instruction, instructional quality, teaching for understanding 


\section{Einleitung}

Ausgehend von einem konstruktivistischen Lernkonzept stellt die Förderung aktiver kognitiver Konstruktionsleistungen der Lernenden ein zentrales Qualitätsmerkmal verständnisorientierten Mathematikunterrichts dar, denn ,je aktiver und selbstmotivierter, je problemlösender und dialogischer, aber auch je bewusster und reflexiver Wissen erworben resp. (ko-)konstruiert wird, desto besser wird es verstanden und behalten (Transparenz, Stabilität), desto beweglicher kann es beim Denken und Handeln genutzt werden (Transfer, Mobilität) und desto bedeutsamer werden die mit dessen Erwerb verbundenen Lernerträge erfahren (Motivationsgewinn, Zugewinn an Lernstrategien, Selbstwirksamkeit)“ (Reusser, 2006, S. 159). Wie aber können aktive Konstruktionsleistungen im Unterricht wirksam initiiert und unterstützt werden bzw. wie lässt sich eine optimale kognitive Aktivierung (Klieme \& Rakoczy, 2008) der Lernenden erreichen? Diese Frage wird zum einen mit Bezug auf methodische Gestaltungsmerkmale des Unterrichts untersucht und diskutiert, zurzeit insbesondere im Zusammenhang mit sogenannten konstruktivistischen oder sozialkonstruktivistischen Lernumgebungen (vgl. z.B. Brophy, 2002). Deren Lernwirksamkeit ist allerdings ebenso umstritten (u.a. Hmelo-Silver, Duncan \& Chinn, 2007; Kirschner, Sweller \& Clark, 2006; Sweller, Kirschner \& Clark, 2007) wie jene des traditionellen, fragendentwickelnden Unterrichts. Jenseits solcher Methodenkontroversen wird zum andern die Position vertreten, dass die kognitive Aktivierung der Lernenden nicht an einen bestimmten Instruktionsansatz gebunden ist (u.a. Klieme, Lipowsky, Rakoczy \& Ratzka, 2006).

Die vorliegenden Datenanalysen auf der Basis von 38 videografierten Unterrichtseinheiten zur Einführung in den Satz des Pythagoras zielen darauf ab, zur Klärung des Zusammenhangs zwischen methodischer Gestaltung und Unterrichtsqualität unter dem Gesichtspunkt der kognitiven Aktivierung beizutragen. Die Auswertungen erfolgten im Rahmen der schweizerisch-deutschen Videostudie „Unterrichtsqualität, Lernverhalten und mathematisches Verständnis“‘1 (Lipowsky, Rakoczy, Klieme, Reusser \& Pauli, 2005a). 


\section{Kognitive Aktivierung durch Gestaltung von Lernumgebungen: Konstruktivistische Instruktionsansätze}

Der Gestaltung (sozial-)konstruktivistischer Lernumgebungen liegt die Idee zugrunde, durch eine bestimmte Unterrichtsinszenierung Lernsituationen zu schaffen, in denen den Lernenden eine aktive(re) Rolle beim Wissensaufbau im Sinne des konstruktivistischen Lernkonzepts zukommt (vgl. Brophy, 2002). Ein typisches Vorgehen besteht z.B. darin, beim Begriffsaufbau das von der Lehrperson straff geführte, fragend-entwickelnde Lehrgespräch zu ersetzen durch eine Kombination von selbstständigem Problemlösen/Explorieren durch die Lernenden mit dem Austausch generierter Ideen in Klassendiskussionen. Untersuchungen solcher Instruktionsansätze basieren häufig auf Fallanalysen oder Designstudien (Brophy, 2006). Während Letztere generell positive Effekte auf den Lernertrag nachwiesen (z.B. Cobb, Wood \& Yackel, 1991; De Corte, Verschaffel, Entwistle \& van Merrienboer, 2003; Stein, Boaler \& Silver, 2003), liegen zur Wirksamkeit konstruktivistischer Instruktionsansätze unter Alltagsbedingungen bisher nur wenige Untersuchungen vor (Brophy, 2006). Im Rahmen unserer Videostudie wurden Zusammenhänge zwischen Instruktionsansatz und Lernerfolg unter Alltagsbedingungen untersucht. Dabei wurden drei als „Inszenierungsmuster“ bezeichnete methodische Vorgehensweisen beim Begriffsaufbau verglichen (Hugener, Pauli \& Reusser, 2007): ein darstellendes, ein problemlösend-entwickelndes und ein problemlösend-entdeckendes Inszenierungsmuster, wobei Letzteres dem oben beschriebenen Beispiel des Begriffsaufbaus im Rahmen konstruktivistischer Lernumgebungen entsprach. Es zeigten sich keine Effekte der Inszenierungsmuster auf den fachlich-kognitiven Lernertrag (ebd.). 


\section{Kognitive Aktivierung als Unterrichtsqualitätsmerkmal}

Ausgehend von der Annahme, dass kognitive Aktivierung und Lernerfolg nicht von einem bestimmten methodischen Vorgehen oder Instruktionsansatz, sondern von tiefer liegenden Qualitätsmerkmalen des Unterrichts und der Lehrer-Schüler-Interaktion abhängen (Hiebert \& Grouws, 2007; Reusser, 2006), wurde in verschiedenen Studien versucht, mit Hilfe von Rating-Verfahren direkt zu erfassen, inwieweit in beobachtetem Unterricht die aktive, konstruktive und diskursive Auseinandersetzung mit dem Lehrstoff unterstützt wird (u.a. Clausen, Reusser \& Klieme, 2003; Klieme, Schümer \& Knoll, 2001; Kunter, 2005). Dieses Verfahren wurde auch im Rahmen unserer Videostudie angewandt. Die Einschätzung der Mathematikstunden hinsichtlich der kognitiven Aktivierung erfolgte durch Expertinnen mit Hilfe eines Rating-Instruments, das in Anlehnung an Kunter (2005) entwickelt worden war (Rakoczy \& Pauli, 2006). Im Gegensatz zu Kunter, die unter Kontrolle der Schulform keine Effekte dieses Merkmals auf die Leistungsentwicklung fand, zeigte sich in unserer Videostudie ein positiver Effekt der kognitiven Aktivierung auf die Entwicklung des konzeptuellen Verständnisses (Lipowsky et al., 2005b). Zudem wurden jene Mathematikstunden, die durch das problemlösend-entdeckende Inszenierungsmuster charakterisiert waren, als kognitiv aktivierender eingeschätzt als die durch ein darstellendes oder fragend-entwickelndes Inszenierungsmuster gekennzeichneten Stunden. Dennoch zeigten sich keine Unterschiede hinsichtlich des Lernerfolgs (Hugener et al., 2007).

Eine Klärung dieser Befunde wird in der vorliegenden Untersuchung mit der Erfassung von zwei unterschiedlichen Dimensionen - Merkmale der Aufgaben und Merkmale des Unterrichtsgesprächs - angestrebt, die beide in der Skala „kognitive Aktivierung“ enthalten sind, und deren Relevanz sowohl theoretisch (Reusser, 2006) als auch empirisch begründet ist. ${ }^{2}$ So verweisen Hiebert et al. (2007) und Stein et al. (2003) auf eine Reihe von Untersuchungen, die positive Effekte eines höheren Anteils kognitiv anspruchsvoller Aufgaben auf den Lernerfolg nachwiesen. Empirisch belegt sind auch positive Effekte 
anspruchsvoller Unterrichtsgespräche, die eine substanzielle Beteiligung der Lernenden zulassen, z.B. als Folge vermehrter Fragen nach Beziehungen und Lösungsmethoden oder des Einforderns von Argumenten oder Erklärungen (vgl. Franke, Kazemi \& Battey, 2007; Schoenfeld, 2006), wobei die diesbezüglichen empirischen Belege allerdings mehrheitlich auf Designstudien zu konstruktivistischen Lernumgebungen basieren (Hiebert \& Grouws, 2007). Eine dritte Dimension, die in der Skala der kognitiven Aktivierung keine zentrale Rolle spielte, aus empirischer (ebd.) wie auch didaktischer Sicht (Aebli, 1983) jedoch hoch relevant erscheint, ist die fachlich-inhaltsbezogene Qualität des Unterrichts (explizite Bearbeitung, Kohärenz, Transparenz der begrifflichen Struktur). Dieser Aspekt wird in den folgenden Auswertungen ebenfalls berücksichtigt.

\section{Fragestellung}

Vor diesem Hintergrund wird untersucht, (1) inwieweit in den videografierten Unterrichtseinheiten zur Einführung in den Satz des Pythagoras systematische Zusammenhänge zwischen der methodischen Vorgehensweise beim Begriffsaufbau und drei Dimensionen eines kognitiv aktivierenden Unterrichts (Auseinandersetzung mit anspruchsvollen Aufgaben, Klassengespräche mit substanzieller Schülerbeteiligung, inhaltliche Kohärenz und Transparenz des Unterrichts) bestehen, und (2) welchen Beitrag diese Merkmale zur Erklärung der Verständnisentwicklung leisten, wobei aufgrund der empirischen Evidenz ein positiver Effekt der drei Qualitätsmerkmale erwartet werden kann. Aufgrund des Forschungsstands wäre zudem zu erwarten, dass die Ausprägung der Qualität nicht vom Instruktionsansatz abhängt.

Um die Fragen zu bearbeiten, wurden die Unterrichtseinheiten aufgrund des Inszenierungsmusters beim Begriffsaufbau in der Einführungsphase in zwei Gruppen aufgeteilt: Einerseits jene Unterrichtseinheiten, die durch einen von der Lehrperson von Anfang an stark geführten Begriffsaufbau gekennzeichnet sind (darstellend, problemlösend- 
entwickelnd), andererseits jene, in denen der Begriffsaufbau dem problemlösendentdeckenden Inszenierungsmuster entspricht.

\section{Methode}

\subsection{Stichprobe und Design}

Es wurden Daten aus dem schweizerisch-deutschen Projekt „Unterrichtsqualität, Lernverhalten und mathematisches Verständnis“" ausgewertet, in dessen Rahmen der Mathematikunterricht in deutschen und schweizerischen Klassen (9. resp. 8. Schuljahr) ${ }^{3}$ der oberen beiden Schulformen (Gymnasium und Real- resp. Sekundarschule) während eines Schuljahrs untersucht wurde (Lipowsky et al., 2005a). In die vorliegenden Analysen wurden 38 Klassen (773 Schüler/innen), einbezogen (Deutschland: 9 Gymnasial- und 10 Realschulklassen; Schweiz: 3 Gymnasial- und 16 Sekundarschulklassen). Gegenstand der folgenden Auswertungen ist eine drei aufeinanderfolgende Mathematikstunden umfassende Unterrichtseinheit zur Einführung in den Satz des Pythagoras, die von einem Vor- und einem Nachtest umrahmt wurde.

\subsection{Erfassung der methodischen Vorgehensweise: geführter vs. explorativ- entdeckender Begriffsaufbau}

Die Identifikation der drei Inszenierungsmuster beim Begriffsaufbau war in früheren Auswertungen mittels einer Kodierung der Sozial- und Lehrformen ${ }^{4}$ und darauf aufbauend mit einem typenbildenden Verfahren vorgenommen worden (Hugener, 2008): Beim darstellenden Verfahren wird der Satz des Pythagoras von der Lehrerperson erklärt, beim problemlösend-entwickelnden Vorgehen wird der Satz anhand einer Problemstellung unter der Führung der Lehrperson im fragend-entwickelnden Lehrgespräch erarbeitet und beim problemlösend-entdeckenden Vorgehen suchen die Lernenden zu einer Problemstellung zunächst selbstständig Lösungswege und/oder generieren Hypothesen, die anschließend im Klassenverband vorgestellt und diskutiert werden. Maßgebend war nur die Phase des 
Begriffsaufbaus, die mit der erstmaligen vollständigen Formulierung des Satzes des Pythagoras endete. Die Gestaltung der nachfolgenden Vertiefungsphase spielte für die

Musterzuteilung keine Rolle. ${ }^{5}$ Für die vorliegende Untersuchung wurden das darstellende und das problemlösend-entwickelnde Verfahren als (von Anfang an), geführtes Vorgehen“ ( $n=$ 19) dem „explorativ-entdeckenden Vorgehen“ $(n=19)$ gegenübergestellt, um an die Methoden-Kontroversen anzuknüpfen, die sich zentral um die Wirksamkeit stärker geführter vs. explorativ-entdeckender Formen des Begriffsaufbaus drehen.

\subsection{Erfassung der Qualitätsmerkmale}

Auseinandersetzung mit anspruchsvollen Aufgaben: Die Erfassung des Ausmaßes an Gelegenheiten zur Auseinandersetzung mit anspruchsvollen Aufgaben wurde aufgrund der Kodierung aller Schülerarbeitsphasen in ,anspruchsvolles“ vs. „repetitives“ Üben (Hugener, Pauli \& Reusser, 2006) vorgenommen. Es wurde der prozentuale zeitliche Anteil anspruchsvollen Übens an der Gesamtzeit aller Schülerarbeitsphasen errechnet. Da dieses Merkmal nicht normal verteilt ist, wurde die Variable bei Verwendung als unabhängige Variable am Median dichotomisiert.

Klassengespräch mit substanzieller Schülerbeteiligung: Indikator ist die durchschnittliche Länge eines Schülerbeitrags in den Klassengesprächen in Anzahl Worten (Lipowsky, Rakoczy, Pauli, Reusser \& Klieme, 2007). Auch dieses Merkmal ist nicht normal verteilt und wurde für die Verwendung als unabhängige Variable mittels Mediansplit dichotomisiert.

Fachlich-inhaltliche Qualität des Unterrichts: Indikator ist die mittels eines RatingVerfahrens erfasste inhaltlich-strukturelle Klarheit der „Theoriephasen“ der Unterrichtseinheit (Drollinger-Vetter \& Lipowsky, 2006). Als Theoriephasen wurden aufgrund der Kodierung der Lehrformen und deren didaktischer Funktion jene Phasen des Unterrichts identifiziert, in 
denen der Satz des Pythagoras entwickelt, formuliert, erklärt oder bewiesen wird. Die Einschätzung der inhaltlich-strukturellen Klarheit erfolgte anhand von 4 einzelnen Ratings (Beispiel-Item: „Kohärenz von Satz und Beweis ist nachvollziehbar“; 1 = niedrige bis $4=$ hohe Ausprägung), aus denen eine Skala mit einer Reliabilität von $\alpha=.88$ gebildet wurde. Die Ratings wurden von zwei Experten je unabhängig vorgenommen und anschließend verglichen; bei abweichenden Urteilen wurde ein Konsens gebildet (für Details siehe Drollinger-Vetter \& Lipowsky, 2006).

\subsection{Lernerfolg und Schülervoraussetzungen}

Im Anschluss an die Unterrichtseinheit absolvierten die Lernenden einen Nachtest, der anhand von 16 Aufgaben das konzeptuelle Verständnis des Satzes des Pythagoras prüfte, indem z.B. für ein gegebenes Dreieck zu entscheiden war, ob der Satz des Pythagoras anwendbar sei (Lipowsky, Drollinger-Vetter, Hartig \& Klieme, 2006). Die Testscores wurden per Item-Response-Theorie berechnet (Modellanpassungswerte der Items zwischen .89 und 1.24; Skala: $M=-0.01, S D=1.29$, Reliabilität .78).

Zur Kontrolle des inhaltsspezifischen Vorwissens wurde ein Vortest herangezogen, der unmittelbar vor der gefilmten Unterrichtseinheit absolviert wurde und anhand von zehn Fragen spezifische Voraussetzungen für das Verständnis von Pythagoras erfasste wie z.B. die Identifikation rechtwinkliger aus einer Reihe abgebildeter Dreiecke (Modellanpassungswerte der Items zwischen 0.92 und 1.03; Skala: $M=0.39, S D=1.27$, Reliabilität .64; detaillierte Angaben bei Lipowsky et al., 2006).

Als weitere Kontrollvariable wurde die kognitive Fähigkeit der Lernenden (Heller \& Perleth, 2000) einbezogen. Da sich die deutsche und die schweizerische Stichprobe hinsichtlich der Jahrgangsstufe sowie hinsichtlich der Verteilung der Schulform 
unterscheiden, wurden bei den Analysen das mittlere Vorwissen und die mittlere kognitive Fähigkeit berücksichtigt.

\section{Ergebnisse}

Um den Zusammenhang zwischen methodischem Vorgehen und Qualität zu untersuchen, wurden Varianzanalysen (unter Kontrolle des mittleren spezifischen Vorwissens und der mittleren kognitiven Fähigkeit) gerechnet.

\section{Tabelle 1 etwa hier einfügen}

Tabelle 1 verdeutlicht, dass es nicht vom methodischen Vorgehen beim Begriffsaufbau (Einführungsphase) abhängt, in welchem Ausmaß die drei erfassten Qualitätsmerkmale über die gesamte Unterrichtseinheit realisiert werden können.

Der Einfluss der Qualitätsmerkmale auf die Entwicklung des Verständnisses des Satzes von Pythagoras wurde mittels Mehrebenenanalysen (Raudenbush, Bryk \& Congdon, 2005) überprüft (vgl. Tab. 2), da die Daten eine genestete Struktur aufweisen. Die Intraklassenkorrelation (ICC) der Leistung im Nachtest beträgt .43. Die beiden Merkmale „Anteil anspruchsvolles Üben an der Schülerarbeitszeit“ und „durchschnittliche Länge der Schülerbeiträge im Klassengespräch“ wurden als dichotome Variablen (dummy-codiert) verwendet. Die Überprüfung möglicher Interaktionseffekte zwischen den beiden Einführungsverfahren (geführter vs. explorativ-entdeckender Begriffsaufbau) einerseits und den Qualitätsmerkmalen andererseits erfolgte mit Hilfe von Multiplikationstermen (methodisches Vorgehen beim Begriffsaufbau x Qualitätsmerkmal). Alle Variablen wurden ztransformiert, so dass sie als standardisierte Regressionsgewichte der Prädiktoren interpretiert werden können.

\section{Tabelle 2 etwa hier einfügen}


Tabelle 2 zeigt, dass sich eine höher eingeschätzte inhaltlich-strukturelle Klarheit der Theoriephasen (Modell 1) und längere Schülerbeiträge im Klassengespräch (Modell 2) positiv auf die Leistung im Nachtest auswirken, nicht aber ein höherer zeitlicher Anteil anspruchsvoller Schülerarbeit an den Schülerarbeitsphasen (Modell 3). Davon abgesehen bestätigen die Ergebnisse, dass die Leistungsentwicklung stark von der individuellen kognitiven Fähigkeit und insbesondere dem spezifischen Vorwissen beeinflusst wird. Die Leistungsfähigkeit der Klasse ist ein bedeutsames Kompositionsmerkmal. Was die Interaktion zwischen methodischer Vorgehensweise und Qualitätsmerkmalen betrifft, deutet sich nur in Bezug auf die durchschnittliche Länge der Schülerbeiträge im Klassengespräch ein schwacher Interaktionseffekt an. Um diesen interpretieren zu können, wurde der Einfluss dieses Merkmals in den beiden Gruppen je einzeln überprüft. Da mit $n=19$ Gruppen eine für Mehrebenenanalysen zu geringe Anzahl von Ebene-2-Einheiten vorliegt, wurde eine Abschätzung des Effekts auf Klassenebene mittels linearer Regression (unter Einschluss von aggregiertem Vorwissen und kognitiver Fähigkeit) unter Vernachlässigung der hierarchischen Datenstruktur vorgenommen. Unter diesem Vorbehalt zeigt das Ergebnis, dass auf Klassenebene längere Schülerbeiträge im Klassengespräch die Nachtestleistung unter der Bedingung des geführten Begriffsaufbaus positiv beeinflussen $(\beta=.37, p=.002)$, während unter der Bedingung des explorativ-entdeckenden Verfahrens kein Einfluss feststellbar ist.

\section{Diskussion}

Die Ergebnisse zeigen, dass sich drei für einen kognitiv aktivierenden Mathematikunterricht und für die Entwicklung des konzeptuellen Verständnisses relevante Qualitätsmerkmale sowohl unter der Bedingung eines durch die Lehrperson geführten als auch eines explorativ-entdeckenden Begriffsaufbaus in der Einführungsphase realisieren lassen. Dies spricht gegen die in aktuellen Kontroversen unterstellte Über- oder 
Unterlegenheit eines bestimmten methodischen Vorgehens und legt die Berücksichtigung methodenunabhängiger Qualitätsmerkmale nahe.

Weitere Auswertungen zeigen, dass das in früheren Analysen mittels hoch inferenter Einschätzungen erhobene Ausmaß der kognitiven Aktivierung positiv mit der durchschnittlichen Länge der Schülerbeiträge korreliert (vgl. auch Lipowsky, Pauli \& Rakoczy, 2008), nicht aber mit der inhaltlich-strukturellen Klarheit der Theoriephasen und dem Anteil anspruchsvollen Übens, was darauf hinweist, dass die Beobachterinnen bei den Ratings besonders auf die Qualität der Klassengespräche achteten und die anderen Aspekte weniger gewichteten.

Neben der durchschnittlichen Länge der Schülerbeiträge im Klassengespräch erwies sich die fachlich-inhaltliche Qualität des Unterrichts, erfasst durch Ratings der inhaltlichstrukturellen Klarheit der Theoriephasen, als bedeutsamer Prädiktor der Entwicklung des konzeptuellen Verständnisses. Dieses Ergebnis steht im Einklang mit den oben dargestellten theoretischen und empirischen Erkenntnissen, wonach der expliziten, durch Kohärenz und Transparenz gekennzeichneten Auseinandersetzung mit dem Lernstoff eine große Bedeutung für das fachlich-kognitive Lernen zukommt. Betrachtet man die Rating-Skalen, die den Einschätzungen der kognitiven Aktivierung zugrunde lagen (Rakoczy \& Pauli, 2006), zeigt sich, dass diese eher auf die kognitive Aktivierung der Lernenden im Allgemeinen ausgerichtet waren und sich nicht zwingend auf die aufzubauende begriffliche Struktur (Satz des Pythagoras) beziehen mussten. So wurde z.B. der kognitive Anspruchs- und Anregungsgehalt von Aufgaben oder Lehrerfragen generell beurteilt (ebd., S. 224-229). Der Befund, wonach ein explorativ-entdeckender Begriffsaufbau zwar mit einer günstigeren Beurteilung der kognitiven Aktivierung (hoch inferente Ratings), nicht aber mit besseren Leistungen im Nachtest verbunden war (Hugener et al., 2007; vgl. Abschnitt 3), könnte demnach auf einem gewissen Bias dieses Rating-Instruments beruhen. Es ist davon 
auszugehen, dass diskursive Klassengespräche und Gelegenheiten zur Auseinandersetzung mit anspruchsvollen Aufgaben nicht an sich, sondern vor allem in Verbindung mit einer hinreichenden fachlich-inhaltlichen Kohärenz und Transparenz des Unterrichts besonders lernförderlich sind. Die Bedeutung des fachlich-inhaltlichen Aspekts wird durch eine kürzlich publizierte Metaanalyse neuerer Befunde zur Unterrichtswirksamkeit unterstrichen: Gelegenheiten zur Ausübung inhaltsbezogener Lernaktivitäten erwiesen sich als wichtiger Prädiktor für den fachlich-kognitiven Lernerfolg und darüber hinaus für die motivationalaffektive Entwicklung (Seidel \& Shavelson, 2007).

Der Interaktionseffekt zwischen Inszenierungsform und Qualität des Klassengesprächs kann als Hinweis auf die in der Literatur oft thematisierte ambivalente Rolle des Klassengesprächs beim fragend-entwickelnden Unterricht (z.B. Leinhardt, 2005; Pauli, 2006) interpretiert werden, sollte jedoch nicht überschätzt werden - zum einen aufgrund der unzureichenden statistischen Absicherung, zum andern aufgrund des verwendeten Indikators. Obwohl sich die Länge der Schülerbeiträge in anderen Untersuchungen als relativ robuster Prädiktor der Leistungsentwicklung herausgestellt hat (z.B. Hiebert \& Wearne, 1993), kann dieser Indikator nur als grobe Annäherung betrachtet werden, nicht zuletzt auch vor dem Hintergrund der oben erwähnten Bedeutung der inhaltlichen Fokussierung. Deshalb werden zurzeit weitere Analysen der Klassengespräche durchgeführt, die u.a. auch inhaltliche Aspekte berücksichtigen.

Einschränkend ist festzuhalten, dass die Ergebnisse auf einer kleinen und nicht zufällig ausgewählten Stichprobe beruhen, die einerseits eine ergebnisbegünstigende Auswahl darstellen könnte und andererseits die Anwendung der statistischen Verfahren einschränkte. Eine weitere Einschränkung besteht darin, dass die Qualitätsmerkmale bezogen auf Theorieelemente, Schülerarbeitsphasen und Klassengespräche während der ganzen Unterrichtseinheit erfasst wurden, während sich die Zuordnung zu den verglichenen 
methodischen Vorgehensweisen des Begriffsaufbaus auf die Einführungsphase im engeren Sinne bezieht. Kompensationseffekte zwischen Einführungs- und Vertiefungsphase sollen in einem weiteren Schritt geklärt werden. 


\section{Literatur}

Aebli, H. (1983). Zwölf Grundformen des Lehrens. Stuttgart: Klett-Cotta.

Brophy, J. (2006). Observational research on generic aspects of classroom teaching. In P. A. Alexander \& P. Winne (Eds.), Handbook of educational psychology ( $2^{\text {nd }}$ ed.; pp. 755-780). Mahwah, NJ: Erlbaum.

Brophy, J. (Ed.). (2002). Social constructivist teaching: Affordances and constraints. Oxford: Elsevier.

Clausen, M., Reusser, K. \& Klieme, E. (2003). Unterrichtsqualität auf der Basis hochinferenter Unterrichtsbeurteilungen. Ein Vergleich zwischen Deutschland und der deutschsprachigen Schweiz. Unterrichtswissenschaft, 31, 122-141.

Cobb, P., Wood, T. \& Yackel, E. (1991). A constructivist approach to second grade mathematics. In E. v. Glasersfeld (Ed.), Radical constructivism in mathematics education (pp. 157-176). Dordrecht: Kluwer.

De Corte, E., Verschaffel, L., Entwistle, N. \& van Merrienboer, J. J. G. (Eds.). (2003). Powerful learning environments: Unravelling basic components and dimensions. Amsterdam: Pergamon.

Drollinger-Vetter, B. \& Lipowsky, F. (2006). Fachdidaktische Qualität der Theoriephasen. In I. Hugener, C. Pauli \& K. Reusser (Hrsg.), Videoanalysen (= Teil 3 der Dokumentation Erhebungs- und Auswertungsinstrumente zur schweizerisch-deutschen Videostudie "Unterrichtsqualität, Lernverhalten und mathematisches Verständnis", hrsg. von E. Klieme, C. Pauli \& K. Reusser, S. 189-205). Frankfurt a.M.: GFPF/DIPF.

Franke, M. L., Kazemi, E. \& Battey, D. (2007). Mathematics teaching and classroom practice. In F. K. Lester (Ed.), Second handbook of research on mathematics teaching and learning. A project of the National Council of Teachers of Mathematics (pp. 225-256). Charlotte, NC: Information Age Publishing/NCTM.

Heller, K. A. \& Perleth, C. (2000). Kognitiver Fähigkeitstest für 4. bis 12. Klassen, Revision (KFT4-12+R). Göttingen: Beltz Test.

Hiebert, J. \& Grouws, D. A. (2007). The effects of classroom mathematics teaching on students' learning. In F. K. Lester (Ed.), Second handbook of research on mathematics teaching and learning (pp. 371-404). Charlotte, NC: Information Age Publishing.

Hiebert, J. \& Wearne, D. (1993). Instructional tasks, classroom discourse, and students' learning in second-grade arithmetic. American Educational Research Journal, 30, 393425.

Hmelo-Silver, C. E., Duncan, R. G. \& Chinn, C. A. (2007). Scaffolding and achievement in problem-based and inquiry learning: A response to Kirschner, Sweller, and Clark (2006). Educational Psychologist, 42, 99-107.

Hugener, I. (in Druck). Inszenierungsmuster im Unterricht und Lernqualität. Sichtstrukturen schweizerischen und deutschen Mathematikunterrichts in ihrer Beziehung zu Schülerwahrnehmung und Lernleistung - eine Videoanalyse. Münster: Waxmann.

Hugener, I., Pauli, C. \& Reusser, K. (2006). Videoanalysen (= Teil 3 der Dokumentation Erhebungs- und Auswertungsinstrumente zur schweizerisch-deutschen Videostudie "Unterrichtsqualität, Lernverhalten und mathematisches Verständnis", hrsg. von E. Klieme, C. Pauli \& K. Reusser). Frankfurt a.M.: GFPF/DIPF.

Hugener, I., Pauli, C. \& Reusser, K. (2007). Inszenierungsmuster, kognitive Aktivierung und Leistung im Mathematikunterricht. Analysen aus der schweizerisch-deutschen Videostudie. In D. Lemmermöhle, M. Rothgangel, S. Bögeholz, M. Hasselhorn \& R. Watermann (Hrsg.), Professionell Lehren - Erfolgreich Lernen (S. 109-121). Münster: Waxmann. 
Kirschner, P. A., Sweller, J. \& Clark, R. E. (2006). Why minimal guidance during instruction does not work: An analysis of the failure of constructivist, discovery, problem-based, experiential, and inquiry-based teaching. Educational Psychologist, 41, 75-86.

Klieme, E., Lipowsky, F., Rakoczy, K. \& Ratzka, N. (2006). Qualitätsdimensionen und Wirksamkeit von Mathematikunterricht. Theoretische Grundlagen und ausgewählte Ergebnisse des Projekts "Pythagoras". In M. Prenzel \& L. Allolio-Näcke (Hrsg.), Untersuchungen zur Bildungsqualität von Schule. Abschlussbericht des DFGSchwerpunktprogramms (S. 127-146). Münster: Waxmann.

Klieme, E., Schümer, G. \& Knoll, S. (2001). Mathematikunterricht in der Sekundarstufe I: "Aufgabenkultur" und Unterrichtsgestaltung. In E. Klieme \& J. Baumert (Hrsg.), TIMSS Impulse für Schule und Unterricht (S. 43-57). Bonn: Bundesministerium für Bildung und Forschung.

Kunter, M. (2005). Multiple Ziele im Mathematikunterricht. Münster: Waxmann.

Leinhardt, G. (2005). Seeing the complexity of standing to the side: Instructional dialogues. Cognition and Instruction, 23, 87-163.

Lipowsky, F., Drollinger-Vetter, B., Hartig, J. \& Klieme, E. (2006). Leistungstests (= Teil 2 der Dokumentation der Erhebungs- und Auswertungsinstrumente zur schweizerischdeutschen Videostudie "Unterrichtsqualität, Lernverhalten und mathematisches Verständnis", hrsg. von E. Klieme, C. Pauli \& K. Reusser). Frankfurt am Main: GFPF/DIPF.

Lipowsky, F., Pauli, C. \& Rakoczy, K. (2008). Schülerbeteiligung und Unterrichtsqualität. In M. Gläser-Zikuda \& J. Seifried (Hrsg.), Lehrerexpertise - Analyse und Bedeutung unterrichtlichen Handelns (S. 67-90). Münster: Waxmann.

Lipowsky, F., Rakoczy, K., Klieme, E., Reusser, K. \& Pauli, C. (2005a). Unterrichtsqualität im Schnittpunkt unterschiedlicher Perspektiven - Rahmenkonzept und erste Ergebnisse einer binationalen Studie zum Mathematikunterricht in der Sekundarstufe I. In H. G. Holtappels \& K. Höhmann (Hrsg.), Schulentwicklung und Schulwirksamkeit.

Systemsteuerung, Bildungschancen und Entwicklung der Schule (S. 223-238). Weinheim: Juventa.

Lipowsky, F., Rakoczy, K., Pauli, C., Reusser, K. \& Klieme, E. (2007). Gleicher Unterricht gleiche Chancen für alle? Die Verteilung von Schülerbeiträgen im Klassenunterricht. Unterrichtswissenschaft, 35, 125-147.

Lipowsky, F., Rakoczy, K., Vetter, B., Klieme, E., Reusser, K. \& Pauli, C. (2005b, April). Quantity and quality of geometry instruction and its short-term impact on students' understanding of Pythagorean theorem. Vortrag auf der Jahrestagung der American Educational Research Association, Montreal, Canada.

Pauli, C. (2006). "Fragend-entwickelnder Unterricht" aus der Sicht der soziokulturalistisch orientierten Unterrichtsgesprächsforschung. In M. Baer, M. Fuchs, P. Füglister, K. Reusser \& H. Wyss (Hrsg.), Didaktik auf psychologischer Grundlage. Von Aeblis kognitionspsychologischer Didaktik zur modernen Lehr-Lernforschung (S. 192-206). Bern: h.e.p.

Rakoczy, K. \& Pauli, C. (2006). Hoch inferentes Rating: Beurteilung der Qualität unterrichtlicher Prozesse. In I. Hugener, C. Pauli \& K. Reusser (Hrsg.), Videoanalysen (= Teil 3 der Dokumentation Erhebungs- und Auswertungsinstrumente zur schweizerischdeutschen Videostudie "Unterrichtsqualität, Lernverhalten und mathematisches Verständnis", hrsg. von E. Klieme, C. Pauli \& K. Reusser, S. 206-233). Frankfurt a.M.: GFPF/DIPF.

Raudenbush, S., Bryk, T. \& Congdon, R. (2005). HLM 6, Hierarchical Linear and Nonlinear Modeling. Chicago: Scientific Software International.

Reusser, K. (2006). Konstruktivismus - vom epistemologischen Leitbegriff zur Erneuerung der didaktischen Kultur. In M. Baer, M. Fuchs, P. Füglister, K. Reusser \& H. Wyss 
(Hrsg.), Didaktik auf psychologischer Grundlage. Von Hans Aeblis kognitionspsychologischer Didaktik zur modernen Lehr- und Lernforschung (S. 151-168). Bern: h.e.p.

Schoenfeld, A. H. (2006). Mathematics teaching and learning. In P. A. Alexander \& P. Winne (Eds.), Handbook of educational psychology ( $2^{\text {nd }}$ ed.; pp. 479-510). Mahwah, NJ: Erlbaum. Seidel, T. \& Shavelson, R. J. (2007). Teaching effectiveness research in the past decade: The role of theory and research design in disentangling meta-analysis results. Review of Educational Research, 77, 454-499.

Stein, M. K., Boaler, J. \& Silver, E. A. (2003). Teaching mathematics through problem solving: Research perspectives. In H. L. Schoen (Ed.), Teaching mathematics through problem solving (pp. 245-256). Reston: NCTM.

Sweller, J., Kirschner, P. A. \& Clark, R. C. (2007). Why minimally guided teaching techniques do not work: A reply to commentaries. Educational Psychologist, 42, 115-121. 


\section{Autorenhinweis}

Dr. Christine Pauli

Universität Zürich

Pädagogisches Institut

Freiestr. 36

CH-8032 Zürich

E-Mail: cpauli@paed.uzh.ch

Barbara Drollinger-Vetter, dipl. Math. ETH

Pädagogische Hochschule Zürich

Kantonsschulstr. 3

CH-8090 Zürich

E-Mail: barbara.drollinger@phzh.ch

Dr. Isabelle Hugener

Universität Zürich

Pädagogisches Institut

Freiestr. 36

CH-8032 Zürich

E-Mail: hugener@paed.uzh.ch

Prof. Dr. Frank Lipowsky

Universität Kassel

Nora-Platiel-Str. 1

D-34109 Kassel

E-Mail: lipowsky@uni-kassel.de 


\section{Fußnoten}

${ }^{1}$ Das Projekt wurde auf deutscher Seite (Leitung: E. Klieme, DIPF, Frankfurt) von der DFG unterstützt (Aktenzeichen KL1057/3), in der Schweiz (Leitung: K. Reusser, C. Pauli, Universität Zürich) vom SNF (Projekt-Nr. 1114-63564.00/1).

${ }^{2}$ Da eine detaillierte Darstellung der empirischen Evidenz zur Bedeutung der Qualitätsmerkmale im Rahmen dieses Beitrags nicht möglich ist, wird hier nur auf zusammenfassende Handbuchartikel verwiesen.

${ }^{3}$ Der Einbezug unterschiedlicher Schuljahre folgt aus der Standardisierung des Unterrichtsinhalts: Die Einführung des Satzes des Pythagoras erfolgt in Deutschland im 9., in der Schweiz im 8. Schuljahr.

${ }^{4}$ Alle herangezogenen niedrig inferenten Kodierungen weisen eine Reliabilität von mindestens $88 \%$ auf (Hugener et al., 2006).

${ }^{5}$ Zwischen den Inszenierungsmustern beim Begriffsaufbau und dem weiteren Verlauf des Unterrichts in der Vertiefungsphase (charakterisiert durch 3 weitere Inszenierungsmuster) bestehen keine systematischen Zusammenhänge (Hugener, in Druck) 
Tabelle 1

Mittelwerte und Standardabweichungen der Qualitätsmerkmale in Unterrichtseinheiten mit „,geführtem “vs. ,explorativ-entdeckendem“ Begriffsaufbau

\begin{tabular}{|c|c|c|c|c|c|c|c|}
\hline \multirow[b]{2}{*}{ Qualitätsmerkmal } & \multirow[b]{2}{*}{ Min - Max } & \multicolumn{2}{|c|}{ Geführter Aufbau ${ }^{a}$} & \multicolumn{2}{|c|}{$\begin{array}{c}\text { Explorativ- } \\
\text { entdeckender Aufbau }\end{array}$} & \multirow[b]{2}{*}{$F(d f=1,34)$} & \multirow[b]{2}{*}{$p$} \\
\hline & & $M$ & $S D$ & $M$ & $S D$ & & \\
\hline $\begin{array}{l}\text { Strukturelle Klarheit } \\
\text { der Theoriephasen } \\
\text { (Ratings, Skala 1-4) }\end{array}$ & $1.13-3.75$ & 2.76 & .72 & 2.63 & .58 & .19 & .67 \\
\hline $\begin{array}{l}\text { Länge der } \\
\text { Schülerbeiträge } \\
\text { (Anzahl der Wörter) }\end{array}$ & $3.85-11.96$ & 5.78 & 1.90 & 6.27 & 1.91 & 1.61 & .21 \\
\hline $\begin{array}{l}\text { Anteil } \\
\text { anspruchsvolles } \\
\text { Üben in Prozent }\end{array}$ & $0-27.84$ & 4.64 & 8.12 & 4.76 & 5.71 & .12 & .73 \\
\hline
\end{tabular}


Tabelle 2

Mehrebenenanalysen zur Vorhersage der Leistung im Nachtest

\begin{tabular}{|c|c|c|c|c|c|c|}
\hline \multirow[b]{2}{*}{ Klassenebene: } & \multicolumn{2}{|c|}{ Modell 1} & \multicolumn{2}{|c|}{ Modell 2} & \multicolumn{2}{|c|}{ Modell 3} \\
\hline & $\beta$ & $p$ & $\beta$ & $p$ & $\beta$ & $p$ \\
\hline Spezifisches Vorwissen & .17 & .02 & .17 & .01 & .18 & .03 \\
\hline Kognitive Fähigkeit & .14 & .01 & .19 & .01 & .22 & .00 \\
\hline Methodisches Vorgehen beim Begriffsaufbau ${ }^{a}$ & .03 & .59 & .05 & .39 & .03 & .65 \\
\hline Strukturelle Klarheit der Theoriephasen & .19 & .00 & - & & - & \\
\hline Method. Vorgehen x strukturelle Klarheit & .04 & .33 & - & & - & \\
\hline Länge der Schülerbeiträge im Klassengespräch ${ }^{b}$ & - & & .15 & .00 & - & \\
\hline Method. Vorgehen x Länge der S-Beiträge & - & & .09 & .07 & - & \\
\hline Anteil anspruchsvolles Üben ${ }^{c}$ & - & & - & & .04 & .46 \\
\hline Method. Vorgehen $\mathrm{x}$ anspruchsvolles Üben & - & & - & & .02 & .77 \\
\hline Individualebene: & & & & & & \\
\hline Spezifisches Vorwissen & .23 & .00 & .23 & .00 & .23 & .00 \\
\hline Kognitive Fähigkeit & .18 & .00 & .18 & .00 & .18 & .00 \\
\hline
\end{tabular}

$\overline{\text { Anmerkungen } .}{ }^{\mathrm{a}} 0$ = geführter Begriffsaufbau, 1 = explorativ-entdeckender Begriffsaufbau; ${ }^{\mathrm{b}} 0=$ Negativgruppe, $1=$ Positivgruppe $(>$ Median $) ;{ }^{\mathrm{c}} 0=$ Negativgruppe, $1=$ Positivgruppe (> Median). $\beta=$ standardisierte Regressionskoeffizienten; - = Effekt nicht modelliert. 